\title{
Incorporación de la Orientación Contemplativa en la Práctica Educativa del Siglo XXI
}

\author{
Incorporation of the Contemplative Orientation in the \\ Educational Practice of the $21^{\text {st }}$ Century
}

Francisco José Leria *

Instituto de Investigación en Ciencias Sociales. Universidad de Atacama

\begin{abstract}
La orientación contemplativa en la enseñanza y el aprendizaje se propone como un enfoque teórico y práctico en respuesta a la necesidad de promover el desarrollo integral del estudiante más allá del énfasis en los procesos de adquisición conceptual y/o el dominio de competencias funcionales. La orientación contemplativa en la enseñanza y el aprendizaje se propone como un enfoque teórico y práctico en respuesta a la necesidad de promover el desarrollo integral del estudiante más allá del énfasis en los procesos de adquisición conceptual y/o el dominio de competencias funcionales. Denominada en el ámbito educativo como Educación Contemplativa, se presenta una investigación descriptivo-teórica con una breve exposición de los contextos que han posibilitado su incorporación y validación en la práctica pedagógica actual: 1) la evidencia empírica que la avala; 2) el rol de las habilidades socio-afectivas y el papel de las prácticas contemplativas para su desarrollo; 3 ) la necesidad de integralidad del currículo contemporáneo; 4)la inclusión educativa; y, 5) las tradiciones ancestrales y sus aportes a las prácticas sociales contemporáneas. Se concluye a la orientación contemplativa dentro de las tendencias actuales para el enriquecimiento de la experiencia educativa y reavivación de la dimensión del Ser en educación, como condición primaria al acto de conocer.
\end{abstract}

Descriptores: Educación alternativa, Práctica pedagógica, Concepto moral, Orientación pedagógica, Actualización de los conocimientos.

The contemplative orientation in teaching and learning is proposed as a theoretical and practical approach in response to the need to promote the integral development of the student beyond the emphasis on the processes of conceptual acquisition and / or mastery of functional competences. The contemplative orientation in teaching and learning is proposed as a theoretical and practical approach in response to the need of promoting the integral development of the student beyond the emphasis on the processes of conceptual acquisition and mastery of functional competences. Named in the educational field as Contemplative Education, a descriptive-theoretical research is presented with a brief exposition of the contexts that have made possible its incorporation and validation in the current pedagogical practice: 1) the empirical evidence that supports it; 2) the role of socio-affective skills and the role of contemplative practices for their development; 3 ) the need for integrality of the contemporary curriculum; 4) the educational inclusion; And, 5) the ancestral traditions and their contributions to contemporary social practices. The contemplative orientation is concluded within current trends for the enrichment of the educational experience and revival of the dimension of Being in education, as a primary condition to the act of knowing.

Keywords: Educational alternatives, Teaching practice, Moral concepts, Educational counselling, Refresher courses.

*Contacto: francisco.leria@uda.cl

ISSN: 1696-4713

www.rinace.net/reice/

revistas.uam.es/reice
Recibido: $\quad 25$ de mayo 2017

$1^{\text {a }}$ Evaluación: 23 de junio 2017

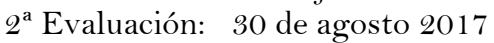

Aceptado: $\quad 3$ de septiembre 2017 


\section{Auscultando una necesidad educativa emergente}

Existe un importante consenso respecto de lo acaecido en el pensamiento científico durante el siglo XX y comienzos del siglo XXI, periodo en el cual se dio paso a una epistemología lejana al concepto de subjetividad, con importantes implicaciones en la ciencia educativa y las prácticas que de ella derivan. En este período la vivencia subjetiva fue puesta en un segundo plano o incluso negada, contribuyendo a la creación de un paradigma científico donde la misma no es la piedra angular y/o participante de la aproximación de estudio, sino un subproducto de elementos constitutivos más simples, y por lo tanto, reducible a los mismos. Fenómeno evidenciado en la denominada "expulsión del sujeto" desde la narrativa científica contemporánea, entró en escena un explícito debilitamiento de la presencia de la subjetividad en el discurso, transformándola en un objeto cuantificable a situaciones fácticas concretas y ajenas al sujeto que conoce. Descubriendo en los extremos la noción del yo-neuroquímico, se tornó innecesario, sino al menos inverosímil, el cultivo de una relación introspectiva del sujeto consigo mismo, y en cuanto ciencia, la subjetividad desde entonces se ha visto amenaza por un proceso de desaparición. Vázquez (2015), parafraseando al filósofo Edgar Morín, enfatiza:

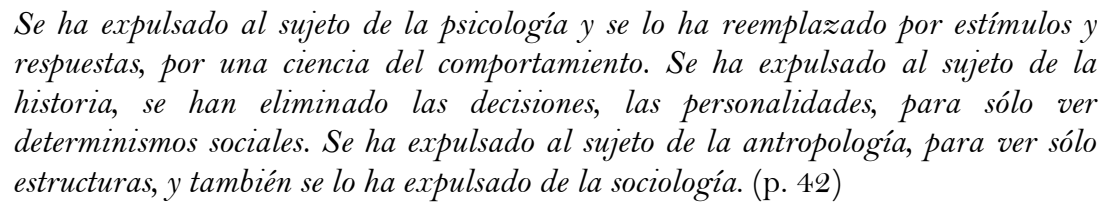

El alcance de este fenómeno socio-histórico por naturaleza tuvo una gran influencia en el pensamiento moderno -y bajo esta mirada- su impacto en la ciencia educativa se caracterizó por la constitución de un objeto de estudio cuya meta final comprende las características universales del estudiante, por sobre sus diferencias, peculiaridades y subjetividades. Esta epistemología denominada positiva, sistema filosófico que resalta el uso del método experimental reduciendo la posibilidad de conocimiento a lo dado a la experiencia sensorio-perceptual, significó el sostén teórico para el establecimiento de una "separación" entre el sujeto investigador y los objetos investigados, y por consiguiente, entre el sujeto que enseña y el sujeto que es enseñando (Matías y Hernández, 2014). Además, generó un fraccionamiento en el estudio de los procesos de enseñanza y aprendizaje desde múltiples pero aisladas dimensiones (ej. lo comportamental, lo cognitivo, lo ambiental, lo social, etc.); produciendo dispersión en la construcción de un objeto epistemológico unificado (Cárdenas et al., 2012). Así, se promovió una mirada disgregada, que derivó en saberes pedagógicos y disciplinares con un objeto de estudio parcializado y desinteresado en las explicaciones teleológicas, impulsando una visión del proceso formativo sin metas finales, valores y sentidos transcendentes, sin la necesidad de colaboración intencional entre el docente y el estudiante y sin la necesidad de la generación de tecnologías educativas afines. Si bien existieron esfuerzos por concebir una educación centrada en los intereses globales y autónomos del educando, por ejemplo, el enfoque clásico de John Dewey (1910); y más tarde en los aportes de la educación humanista y los modelos del aprendizaje significativo; estos intentos se vieron trastrocados por el impacto y amplio uso de las teorías positivistas provenientes de la escuela del análisis funcional de la conducta. Estos planteamientos dejaron a un lado importantes dimensiones y variables que hoy en día se sabe poseen una función crucial en el aprendizaje y desarrollo psicológico; y si bien no es discutible el impacto y aportes 
que este enfoque epistémico ha tenido en la ciencia educativa, existe acuerdo respecto de sus consecuencias, implicando una concepción particular y más bien estrecha del proceso de educar (Segovia, 2013).

Con el avanzar de las décadas y la adopción de una perspectiva postmoderna en educación, se consideró que la explicación del acto educativo y el fenómeno de conocimiento implícito, no es suficiente desde los aspectos universales e invariables del estudiante, sino que la importancia debía radicar en una actitud de responsabilidad individual en el proceso de aprendizaje y el sentido último del proceso formativo. Estos planteamientos adquirieron la denominación de enfoque socio-constructivista, entreluciendo el papel de la variable subjetiva y su reconocimiento cada vez más palpable en el saber pedagógico. No obstante, en el tiempo y junto a sus enormes, actuales e innegables aportes, apareció otro fundamentalismo, de tributar a la lógica de la reproducción del sistema que lo domina, y bajo su tónica subjetivista y liberadora, se afirma es tecnocrático y mejor se ajusta al modelo neoliberal (Mazorco, 2010). Si bien este enfoque ha generado un inmenso aporte al diseño y práctica pedagógica (Serrano y Pons, 2011), ha causado también una proliferación poco fundamentada del mismo, junto al continuo privilegio por la formación cognitiva y la exigencia disciplinar (Duque y Ramírez, 2012). Constituyéndose en un avance en torno a la reincorporación de la subjetividad, este abrieron las puertas a nuevas variables de importancia para la calidad y profundidad del proceso formativo, haciendo propicio la investigación de las variables propias del estudiante asociadas a mayores niveles de eficacia y eficiencia en su desempeño cognitivo en los espacios reales de estudio, junto a otras variables asociadas a una mayor satisfacción consigo mismo y la vida (Cejudo, López-Delgado y Rubio, 2016).

En sincronía con lo planteado por Taylor (2007) y su concepto del cambio masivo de la subjetividad en la cultura moderna; las distintas orientaciones pedagógicas han incorporado elementos propios a la vivencia de la persona, por ejemplo: el bienestar, las emociones, la creatividad, la expresión artística, la exploración ambiental, la espiritualidad, entre otras; derivando en una perspectiva multipedagógica, hacia modos de vida en los cuales los sujetos-estudiantes construyan una mayor sintonía percibida con su propia experiencia y entorno. Estas perspectivas se configuraron en plataformas de apoyo para el cambio epistémico que ha venido ocurriendo en el presente siglo, en una época post-secular, donde las demarcaciones entre religión, espiritualidad y sus interrelaciones se han hecho difusas y, por lo tanto, más presentes en la cotidianidad de las personas.

\section{La expansión de la experiencia educativa}

El carácter temporal de toda verdad científica ha derivado en la necesidad por enfrentar las consecuencias y vacíos derivados del predominio de los enfoques epistemológicos que clásicamente han nutrido a la pedagogía; y que la han privado da las variables propiamente subjetivas, lejanas al concepto de desempeño, competencia y/o rendimiento. En la búsqueda de devolver la variable humana al proceso de aprender (Bisquerra y Pérez-Escoda, 2012); se diagnosticó primariamente un vacío en la experiencia educativa debido a la excesiva formación con énfasis en lo cognitivo, racional y reflexivo. Se propuso así la necesidad de desarrollar modelos pedagógicos que resultaran en capacidades y habilidades acordes con el nuevo escenario económico-social, que respondieran a las necesidades reales que los sujetos requieren en su entorno inmediato, 
e integraran, como lo sugiere declarativamente el informe Delors (1996): "al desarrollo global de cada persona: cuerpo y mente, inteligencia, sensibilidad, sentido estético, responsabilidad individual, espiritualidad" (p. 99). Consecuentemente, las habilidades y/o competencias emocionales entraron en escena dentro de las estrategias para el desarrollo de los aprendizajes (Bisquerra y Pérez-Escoda, 2012), transformándose en el tiempo en un grupo de enfoques pedagógicos, amplio e integral, denominados: Pedagogías centradas en el corazón y/o heartfulness (Daugherty, 2014); enfoques que relevan el desarrollo de la vivencia emocional como gestora de los aprendizajes y desarrollo psicosocial.

Generándose apertura por el enriquecimiento del enfoque tradicional en educación, a finales del siglo XX se da cabida a la incorporación de nuevas variables a los modelos pedagógicos, logrando el punto culmine con la popularización de los modelos de las inteligencias múltiples (Gardner, 1987), la inteligencia práctica (Sternberg, 1985), la inteligencia emocional (Goleman, 1996) y el modelo de la competencia emocional (Mayer y Salovey, 1997). Los mismos propusieron miradas alternativas por sobre el tradicional predominio de las variables cognitivas, incorporando otras habilidades que hacen posible una persona se desenvuelva con éxito en la vida, abriendo así paso a la aceptación de las variables no-cognitivas como agentes, sino primarios al menos esenciales, del éxito en los aprendizajes. Esta apertura ha tenido el propósito compartido de redefinir la mirada de la educación a dominios del aprendizaje que representen una integralidad en el enfoque, con un carácter teórico, pero por sobre todo vivencial, integrando aspectos que el currículo tradicional ha dejado históricamente fuera y teniendo en común una visión multi-pedagógica (Paymal, 2014). Los cambios y trasformaciones por una expansión curricular de la experiencia educativa han además incluido un factor transdisciplinario, alineándose con las tendencias de la ciencia basada en la evidencia para estrechar la brecha entre la investigación y la práctica educativa (Páramo y Hederich, 2014). Tal tendencia fortaleció la necesidad de incorporar los hallazgos de varias de las disciplinas científicas que tradicionalmente nutren a la pedagogía, como la Psicología, Sociología, Antropología y Neurociencia; por su aporte sustantivo a la calidad y eficacia del diseño curricular, la didáctica y la evaluación de los aprendizajes. En este sentido, el desarrollo científico ha reunido un considerable cúmulo de evidencia, que contribuye a la validación no solo de las variables cognitivas, sino de aquellas en que tal componente no es primario y/o exclusivo (ej. las emociones), como aspectos esenciales al proceso de enseñanza y aprendizaje. En la última década se ha observado un entusiasmo respecto de los alcances y aplicación de esta evidencia científica en los procesos formativos. Por ejemplo, en el caso de la neurociencia educativa y la facilitación de los procesos de aprendizaje e influencia de los procesos emocionales, siendo en muchos sentidos sus aportes prácticos orientados al saber disciplinario del profesional-educador; al punto de considerar difícil de concebir un enfoque pedagógico sin incluir sus aportes (Ortiz, 2009; Puebla y Talma, 2011).

Distintas corrientes de pensamiento educativo no tradicional han surgido con el propósito por redefinir la mirada a la educación, desde el sustantivo al verbo, a cuatro ámbitos o dominios del aprendizaje que representan una integralidad en el enfoque con un carácter teórico, pero por sobre todo vivencial: aprender a aprender; aprender a hacer; aprender a vivir juntos, y; aprender a Ser; sintetizado en lo siguiente (Paymal, 2008):

Una tendencia general al entrar en este nuevo ciclo, es que todo movimiento educativo del tercer milenio apunte a un desarrollo integral y holístico del ser 
humano en todos sus niveles, "aperturando" y "ensanchando" a un nivel superior de conciencia, que debe natural e intrínsecamente nacer del mismo corazón del ser humano. Por eso, de aqui en adelante, se debería escribir "Educación" con E mayúscula, ya que se trata de elevarnos como seres humanos a una octava superior de conciencia. (p. 3 )

Desde entonces han fructificado una serie de modelos y enfoques pedagógicos que comparten el propósito de integrar los aspectos que el currículo tradicional ha dejado históricamente afuera, particularmente las emociones en el proceso formativo, teniendo en común una visión multi-pedagógica, más integral, que intenta generar una síntesis entre pedagogía, ciencia y otras dimensiones vitales de la experiencia humana como la espiritual. Se pueden destacar de estos modelos por mencionar solo algunos: la Pedagogía Waldorf, el método Montessori, el método Kilpatrick basado en la acción, la Pedagogía liberadora de Paulo Freire, el método Idejo, el método Kurmi Wasi un colegio para la diversidad, la educación biocéntrica, la biodanza, la pedagogía para la vida, la Educación para la Paz, la Pedagogía 3000, o Bosquescuela, la escuela Francisco Varela, entre muchos otros (Paymal, 2014).

Dentro de estas propuestas no tradicionales nacidas al alero del siglo XXI, se ha observado manifestado una "revolución silenciosa" (Martínez Ruíz, 2015), para referirse a la incorporación del desarrollo de la atención consciente como un propósito y objetivo educativo, que, al tornarse en un enfoque pedagógico consolidado, adquiere la denominación de Educación Contemplativa.

\section{La orientación contemplativa en la enseñanza y el aprendizaje}

En el contexto descrito surge la orientación contemplativa en la enseñanza y el aprendizaje y/o educación contemplativa, disciplina de reciente aparición, pero larga existencia y tradición. Corresponde a un enfoque secularizado que promueve la potenciación del desarrollo psicofísico y socioemocional, a través de experiencias educativas que sitúan a la consciencia, las emociones y el cultivo del silencio en el centro de su quehacer pedagógico. A veces denominada meta-pedagogía o pedagogía del proceso (Ergas, 2015a), se nutre de los aportes de una serie de áreas científicas colindantes, esperando enriquecer la práctica educativa con procedimientos validados científicamente, con un carácter preventivo universal y potenciador del desarrollo global del sujeto. Esta orientación intenta dar respuesta a la necesidad de promover el aprendizaje en un sentido amplio, como una forma de reconocimiento explícito de todos los espacios en el que ocurre (García y Klein, 2014). Además, se hace cargo de los vacíos percibidos en el aprendizaje de la buena convivencia y trato, relevando la necesidad educativa por la existencia de un Aula de Convivencia (Oña Cots y García Gálvez, 2016); y promoviendo así una cultura de la paz y consciencia en los centros educativos.

La orientación contemplativa en la enseñanza y el aprendizaje promueve una comprensión profunda de las dimensiones internas de sí mismo, para alcanzar una percepción más plena de la realidad individual y social en la que el estudiante se desenvuelve (Chano, 2012). Uno de sus fundamentos epistemológicos hace referencia al concepto de alfabetización noética, que alude según Paymal (2014): 
Educar al pequeño (estudiante) en la Conciencia y llegamos entonces al tercer presupuesto tridimensional, que agrega la categoría de dimensión noética, la conciencia de la conciencia, la mente contemplativa, el Tao de la Educación. A partir de esta visión el ser humano es un compuesto somático, psíquico y noético (proveniente del griego, nous, que significa (...). ¿Cómo educar la dimensión noética? Con los caminos milenarios de despertar, de las tradiciones sapienciales, orientales y occidentales, naturalmente. A través de las vías meditativas de las antiguas escuelas de sabiduría (p. 251)

Se define a esta orientación como un conjunto de prácticas pedagógicas que tienen como objetivo: "el crecimiento personal y transformación social, a través del cultivo de la consciencia en un contexto ético relacional" (Roeser y Peck, 2009, p. 119), y/o comprender cualquier acto de enseñanza basado en una deliberación del profesor al pedir de sus estudiantes el responder al aquí y ahora de la experiencia de aprendizaje. Ergas (2015a) complementa esta definición con lo siguiente:

El andamio meta-pedagógico de las prácticas contemplativas involucra
deliberadamente a los estudiantes en el aquí y ahora. Al hacerlo contemplativo
prácticas están introduciendo una deliberación curricular alternativo; uno que
desafía, inevitablemente, el concepto mismo de "curriculum" como un "curso de
estudio" y sus implicaciones para nuestra concepción de la vida - significado y donde
deberíamos buscarlo (aquí y/o por ahi). (p. 3)

Las condiciones que han permitido la aparición de la orientación contemplativa en la enseñanza y el aprendizaje se circunscriben a una serie de profundos cuestionamientos respecto del acto de conocer y una de las principales dificultades percibidas en los modelos pedagógicos actuales, concerniente a la desmesurada orientación al qué pensar; por sobre el cómo pensar. Históricamente ha existido una visión distal del proceso educativo, la cual implica una ausencia de formas de observación de sí mismo y los patrones conductuales que le subsiguen, por sobre el tradicional enfoque en la adquisición de contenido conceptual, desarrollo de habilidades y/o competencias funcionales. Reconociendo la importancia del pensamiento como el axis del proceso formativo, y a su vez, su carácter en gran parte improductivo, autores clásicos en educación han señalado: "deberíamos admitir, incluso respecto de nosotros mismos, que (el pensamiento) es probable se mantenga en la distancia de la insignificante e inconsecuente fantasía ociosa y la esperanza insustancial” (Dewey, 1910, p. 14). La noción de pensamiento improductivo, divagación mental y/o en idioma inglés de mindwavering, hace referencia a un proceso psicológico con una importante incidencia en el desempeño cognitivo y que, sin embargo, no ha sido abordado abiertamente por los modelos educativos y metodologías actuales (Ergas, 2015b) en consideración de la evidencia empírica (Seli et al., 2017), por ejemplo, por su papel inhibidor de la metacognición (Kok y Singer, 2017), y/o por el contrario, facilitador del uso del lenguaje y autoanálisis (Bastián et al., 2017).

La teoría educativa que subyace a la orientación contemplativa en la enseñanza y el aprendizaje presupone que las capacidades de la atención voluntaria sostenida, el equilibrio emocional, la comprensión emocional y la compasión son susceptibles de desarrollo a través de la práctica (Zajonc, 2013). El ejercicio y/o práctica de la contemplación, condición sine qua non a la orientación contemplativa, hacen referencia al desarrollo de la habilidad de llevar una cierta calidad, sensibilidad y consciencia a la capacidad atencional del estudiante en la cotidianidad diaria de sus espacios de aprendizaje. Tradicionalmente han sido diseñadas con el propósito de calmar la mente y redireccionarla de su habitual rumiación repetitiva y encubierta al cultivo de un darse 
cuenta de mayor profundidad y concentración, para derivar -según autores como Hart (2004)-, a un razonamiento de una cualidad más cercana a la no-linealidad. La literatura contemporánea refiere comúnmente a esta habilidad de observación y/o contemplación mental, como mindful awareness, mindfulness y/o en idioma castellano: atención plena. Estas habilidades son comúnmente definidas como: "la capacidad de poner una atención deliberada en el presente, momento a momento" (Kabat-Zinn, 2003, p. 145); "la tendencia a ser altamente conscientes de las experiencias internas y externas en el contexto de una postura de aceptación y no enjuiciamiento hacia esas experiencias" (Cardaciotto et al., 2008, p. 205). De acuerdo a la evidencia científica en el área, el ejercicio sistemático de la contemplación (p. ej., a través de la meditación, arte, danza, alimentación, visualización, escucha, y rituales conscientes; entre muchas otras actividades y experiencias), resulta en el fortalecimiento de una serie de habilidades centrales para el proceso formativo del sujeto desde temprana edad; y su impacto abarca una serie de funciones cognitivas y habilidades socioafectivas generales y específicas, existiendo una amplia literatura al respecto de programa y/o actividades extra programáticas para el desarrollo de habilidades contemplativas en sus distintas formas, enfoques y/o manifestaciones. Este impacto se puede resumir en la figura 1.

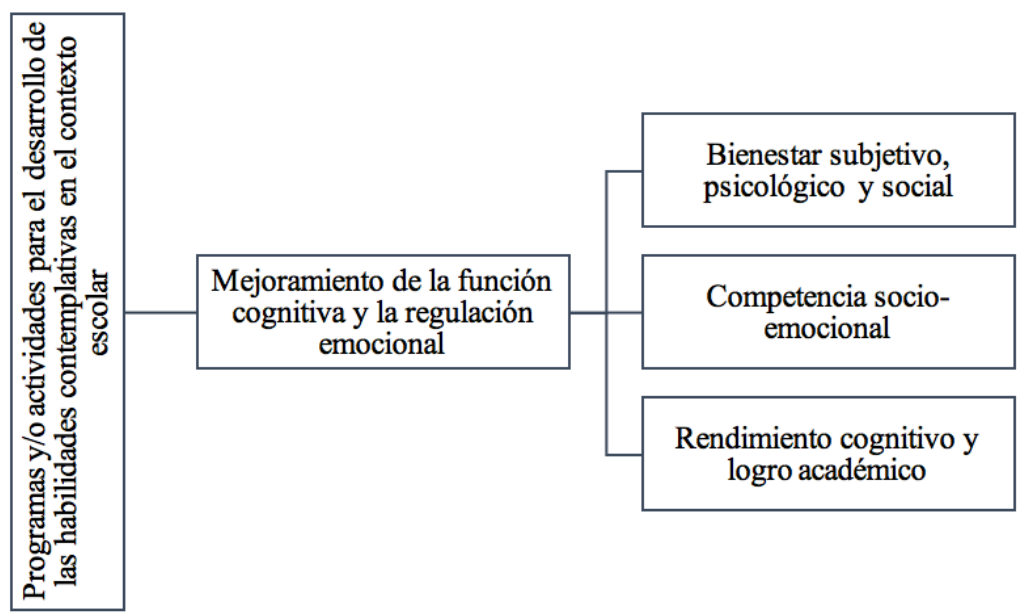

Figura 1. Principales áreas de impacto de las prácticas contemplativas en el estudiante Fuente: Elaboración propia a partir de Waters, Barsky, Ridd y Allen (2014).

El denominado giro contemplativo en educación (Ergas, 2014), se ha conformado en uno de los aportes para responder a la necesidad destacada por algunos autores de re encantar el mundo (Berman, 1987), o re-encantar al sujeto con su subjetividad (Cajigas, 2007); ofreciendo una mirada fresca y transcendente al proceso de educar, más allá de la adquisición de contenidos y/o desarrollo de competencias funcionales vinculadas a las necesidades el contexto socioeconómico actual. Propone formas para la vinculación activa y efectiva del sujeto consigo mismo, como condición esencial para un desarrollo psicológico balanceado, como una condición promotora para el desarrollo de las capacidades necesarias para la consecución exitosa de los aprendizajes y satisfacción con la vida. 


\section{Contextos de emergencia para la incorporación de la orientación contemplativa en la enseñanza y el aprendizaje en la práctica educativa del siglo XXI}

Existen varios contextos científicos y socioculturales que contribuyen a la aparición, validación y consolidación de esta orientación pedagógica en el contexto educativo contemporáneo, los cuales se han conjugado en condiciones propicias para su incorporación y promoción entre los actores educativos interesados en formas y enfoques pedagógicos novedosos e innovadores, ante todo validados empíricamente, para el desarrollo del aprendizaje y buena convivencia en los espacios educativos. A continuación, se describen algunos de los contextos más relevantes que han posibilitado la inclusión de la contemplación y desarrollo de la atención plena en el contexto escolar (figura 2).

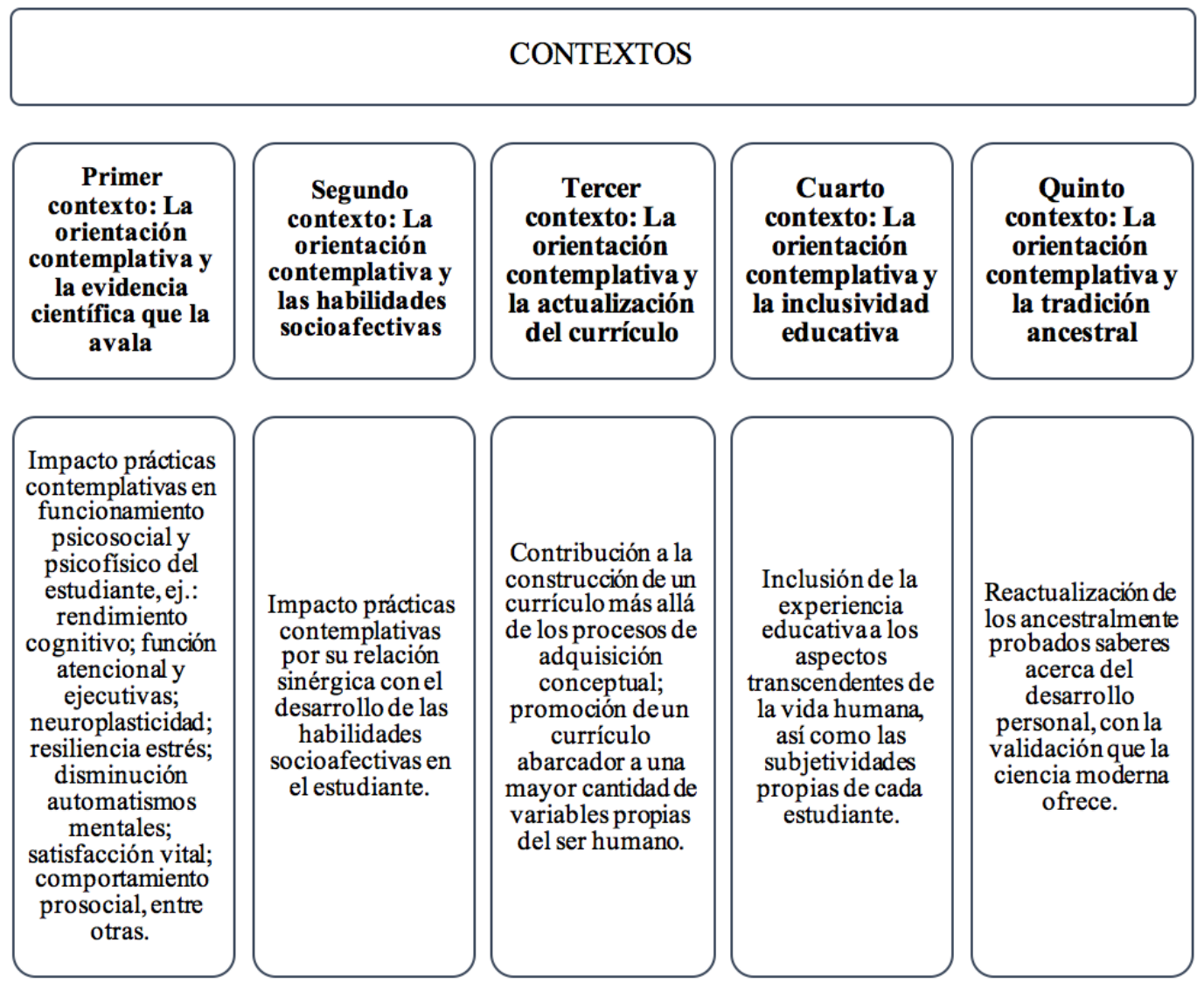

Figura 2. Resumen de los contextos de emergencia para la incorporación de la orientación contemplativa en la enseñanza y el aprendizaje en la práctica educativa del

$$
\text { siglo XXI }
$$

Fuente: Elaboración propia.

\subsection{Primer contexto: La orientación contemplativa y la evidencia científica que la avala}

La evidencia científica releva el aporte de las prácticas contemplativas en el funcionamiento de varias áreas constitutivas del sujeto: neurofisiológicas, cognitivas y 
psicosociales, que las hacen temática de interés del campo educativo. Ha sido justamente esta evidencia el sustento para su inclusión en el diseño curricular basado en la evidencia, junto a la creación de actividades e intervenciones escolares a fines. A la fecha se pueden contar en cientos los estudios que han precisado el impacto, características y proyecciones que las prácticas contemplativas poseen; y que al presente siguen siendo objeto de investigación activa por la comunidad científica del área. Existiendo algunas líneas recurrentes de investigación, a modo de ejemplo, se destacan: mejoramiento del rendimiento cognitivo (Zenner, Herrnleben y Walach, 2014), potenciación del descentramiento cognitivo (Shepherd et al., 2016), disminución de la divagación mental (Trousselard et al., 2014), mejoramiento de la función atencional (Thomas y Atkinson, 2016), mejoramiento de las funciones ejecutivas (Teper, Segal y Inzlicht, 2013), impacto en la neuroplasticidad encefálica (Kirk, Fatola y Romero, 2016), mejoramiento del estado basal del encéfalo (Tomasino, Chiesa y Fabbro, 2014), resiliencia al estrés (Zenner, Herrnleben y Walach, 2014), potenciación de las habilidades socioafectivas (Remmers, Topolinski y Koole, 2016), integración del conocimiento de sí-mismo (Ghasemipour, Robinson y Ghorbani, 2013), disminución de los automatismos mentales (Mañas et al., 2014), impacto en la satisfacción vital (Burack, 2014), promoción del comportamiento prosocial (Flook et al., 2015), y facilitación de la conectividad social (Kok y Singer, 2017); entre muchos otros estudios e investigaciones que evidencian el aporte de las prácticas contemplativas el desarrollo psicofísico del sujeto.

Son estos resultados los que han despertado el interés por su aplicabilidad en los contextos reales de enseñanza y aprendizaje, promoviendo la construcción de programas e intervenciones orientadas al desarrollo de las habilidades contemplativas en el ámbito escolar temprano, primario, secundario y universitario (Barbezat y Bush, 2014; Jennings et al., 2013; Langer et al., 2015; Langer et al., 2017; Meiklejohn et al., 2012). Se cuentan entre los programas más conocidos y validados que incorporan directamente la variable contemplativa y/o abarcan el desarrollo de la consciencia y el fortalecimiento de la función atencional: Aulas felices, Collaborative for academics social and emotional learning, Cultivating awareness and resilience in education, Mindful awareness practices, Mindfulness in schools Project, Mindfulness curriculum for 11-18 year old in schools, Mindfulness ambassadors, Mindful schools organization, Mindful life, The association for contemplative mind in higher education, y The school-based meditation model, entre muchos otros.

\subsection{Segundo contexto: La orientación contemplativa y las habilidades socioafectivas}

Existe asentimiento entre los especialistas respecto del papel de la dimensión socioemocional en educación y su papel como eje y gestor de los procesos de enseñanzaaprendizaje y buena convivencia (Durlak et al., 2011) específicamente por su impacto en el desempeño académico y bienestar subjetivo (Peña, Mikolajczak y Gross, 2015), en la neurofisiología del organismo (Immordino-Yang y Faeth, 2010), específicamente por su efecto promotor del aprendizaje procesal no orientado a tareas específicas (Galante et al., 2014) o, por ejemplo, por el impacto positivo en el manejo de las emociones disruptivas en situaciones sociales (Roeser y Peck, 2009). Las evidencias se muestran a favor del desarrollo de las emociones en el contexto de aprendizaje (Schutte, Malouff y Thorsteinsson, 2013); y hoy existen hallazgos respecto de su relación sinérgica y complementaria con las prácticas contemplativas, por ejemplo, en su papel mutuo en la 
percepción subjetiva de satisfacción con la vida, en contraposición al estrés e insatisfacción (Wang y Kong, 2014).

Dentro de las habilidades de índole cognitiva que han sido ampliamente estudiadas y se afirma promueven el aprendizaje y la gestión de las emociones, destaca la capacidad atencional, gestión de la atención y/o control atencional. Estudios en neurociencia señalan a la conciencia y el fortalecimiento de la atención, como aspectos esenciales para un adecuado manejo del mundo emocional (Damasio, 2000); considerando que la capacidad atencional puede ser entrenada; y su desarrollo permite acceder a un mayor control voluntario sobre los estados emocionales, lo que resulta -entre otras habilidades- en un desempeño social más equilibrado (González et al., 2011), y en el rendimiento cognitivo y logro académico (Chiesa, Calati, y Serretti, 2011; Garland et al., 2015; Brockman et al., 2017). Esta capacidad esencial para el desarrollo socioemocional ha sido validada por varios de los modelos de la inteligencia y competencia emocional, con gran impacto en la práctica educativa del presente siglo. Por ejemplo, los planteamientos de Goleman (1996) señalan a la conciencia y atención en uno mismo como la habilidad de entrada para el desarrollo de la inteligencia emocional y todas sus subcompetencias. Otro importante autor, Bar-On (1997, citado por García y Giménez, 2010), enfatiza que la comprensión emocional de sí mismo tiene un papel esencial en la capacidad de sujeto de comprender sus sentimientos y emociones, diferenciarlos y conocer los motivantes y condicionantes de los mismos. Uno de los más conocidos modelos de la inteligencia emocional en la academia, Mayer, Caruso y Salovey (2008), enfatiza a la percepción emocional y/o habilidad para identificar las emociones en sí mismo y en los demás, como una habilidad esencial y base para el desarrollo de la inteligencia emocional. Otros modelos destacan de una u otra manera las mismas habilidades atencionales y conciencia de uno mismo como aspectos centrales en el manejo de la vivencia emocional en el sujeto (García y Giménez, 2010).

A lo largo de este texto se citan varios estudios que han mostrado el efecto de los programas para el desarrollo de las habilidades contemplativas en el control atencional y la vivencia socioemocional, situando en la actualidad a las prácticas contemplativas (ej. control atencional, silencio, sensibilización a la experiencia corporal; entre otras), como una de las estrategias más validadas empíricamente para su desarrollo (Black, Milam y Sussman, 2009; Randal, Pratt y Bucci, 2015; entre muchos otros).

\subsection{Tercer contexto: La orientación contemplativa y la actualización del currículo}

La aspiración por la calidad educativa ha promovido la construcción del currículo desde una pluralidad de dimensiones, que sea válido para una sociedad y época histórica (Díaz, Carmona y Nadia, 2010). Para este cometido, se ha afirmado el currículo debe ir más allá de la exclusiva atención a los procesos de adquisición conceptual y/o desarrollo pragmático de competencias funcionales, sino poseer una serie de características amplias y multidimensionales, que finalmente le permitan constituirse en un real aporte a la calidad de los procesos formativos. El diseño curricular debe -en este sentido- facilitar un proceso educativo global, abarcando la mayor cantidad de variables propias del ser humano: individuales y grupales; teóricas y prácticas; cognitivas y emocionales; pragmáticas y transcendentes. Algunos autores destacan la necesidad de una metodología diversificada, facilitadora de la calidad para todos los participantes de la comunidad educativa, junto a contenidos debidamente seleccionados para prevenir el 
exceso de información existente en la época actual y su efecto iatrogénico en el control atencional del estudiante (Casanova, 2012).

La orientación contemplativa ha intentado enriquecer las perspectivas del currículo con una mirada epistemológicamente amplia y técnicamente menos circunscrita "al hacer" por sobre el Ser, incorporando un carácter transformativo, más allá de la circunscripción racional de la experiencia de aprendizaje (Palmer, Zajonc y Scribner, 2010). Desde esta perspectiva, el abogar por el currículo integral descubre la necesidad por la inclusión de saberes disciplinares que generen una experiencia significativa y enriquecida en la persona que se educa, reconociendo en la contemplación un rol primario para el acceso a tal experiencia. Ferrer y Sherman (2009) señalan que para un currículo de amplio y no reductivo se deben incluir al menos tres factores, a los cuales les debe corresponder en un sentido transversal, actividades y experiencias educativas atingentes y frecuentes, a saber: 1) el factor holístico; 2) el factor transformativo, y; 3) el factor de integración. Un currículo solamente orientado a los elementos cognitivos, deportivos y/o artísticos, carecería de este sentido amplio; y, por lo tanto, no facilitaría el conocimiento de otras áreas (factor holístico); privaría de una experiencia significativa y transcendente de las mismas (factor transformativo); e impediría una experiencia global unitaria, ecológica e integral del estudiante consigo mismo y su entorno de vida (factor de integración). La orientación contemplativa busca articular el currículo con los principios del procedimiento (Martínez Bonafé, 1993, como se citó en Álvarez, 2012), abogando por un diseño curricular de orden didáctico que concrete: "en la actividad teórico-práctica del profesor el puente entre el proyecto curricular como posibilidad y las realidades concretas del currículo en acción” (p. 43). La orientación contemplativa aporta a la superación del quiebre muchas veces existente entre la teoría y la práctica, particularmente, cuando la intención es introducir metodologías pedagógicas orientadas al desarrollo socioafectivo en el aula.

\subsection{Cuarto contexto: La orientación contemplativa y la inclusividad educativa}

La validación creciente de la inclusividad educativa en las políticas públicas ha abierto caminos más explícitos para la valoración de la particularidad y perfil propio de cada estudiante, sus contextos socioculturales, identidades y motivaciones, como elementos importantes a considerar dentro de las dinámicas de aprendizaje. De una noción restringida se dio paso a una más amplia que considera no sólo a los niños, niñas y jóvenes con necesidades especiales, sino a otros grupos tradicionalmente marginados de los sistemas escolares formales -y en un sentido rector- el concepto se empezó a concebir como un principio general para reforzar la educación para el desarrollo sostenible del aprendizaje a lo largo de toda la vida (UNESCO, 2008, p. 3). Si bien existe acuerdo que la inclusión educativa debe estar comprometida con una agenda de justicia y equidad, en la práctica dista de sus objetivos originales, existiendo una brecha entre lo que se declara y lo que se realiza (Leria et al., 2016).

La posibilidad de responder curricularmente a la revalorización del sujeto, sus diferencias y peculiaridades, ha incurrido en la construcción de un currículo que incorpore experiencias educativas que validen a la diferencia entre los estudiantes, la promuevan y mejor satisfagan las necesidades actuales y contextualizadas de las personas, no impuestas desde afuera de su contexto social y local primario (Segovia, 2013). Se espera garantizar el acceso y la permanencia exitosa de todos los estudiantes 
en el sistema escolar, por medio de prácticas curriculares diversificadas y ajustadas a los diferentes ritmos e intereses de aprendizaje.

Dentro de los debates que la temática de la inclusividad ha generado, resalta la necesidad de incluir en la experiencia formativa, los aspectos transcendentes de la vida humana, dentro de las nuevas formas de conocer propias a los movimientos por la integración en el aprendizaje (Murray, 2009). En este camino, la espiritualidad en el aula ha salido de sus confines dogmáticos por un enfoque más pragmático y vivencial, en la cual el factor transformativo adquiere una mayor relevancia. La orientación contemplativa en la enseñanza y el aprendizaje ofrece una robusta ontología, un amplio rango de métodos y una ética inclusiva, que va más allá de la dinámica moderna del uso-costo-beneficio, que viene a constituirse en un aporte al concepto de inclusividad educativa. Si bien tema de menor urgencia frente otras problemáticas que aquejan a los países en torno a la inclusión; la discusión continúa respecto de la necesidad de incorporar una experiencia transcendente y transformativa en el sujeto que se educa (Lynn, 2010).

\subsection{Quinto contexto: La orientación contemplativa y la tradición ancestral}

Los movimientos por la revaloración de las tradiciones ancestrales y espirituales del mundo han permeado y realizado importantes aportes a las prácticas sociales y modos de vida contemporáneos. Han surgido del acercamiento entre las culturas occidentales y orientales, buscando revitalizar las formas tradicionales de acercamiento del sujeto a su interioridad, con prácticas activas de autoexploración, cuidado y conocimiento de sí mismo. Observado este movimiento muchas veces dentro de la noción de neochamanismo, ha sido inspirado en una filosofía de vida enfilada a la restitución de la dimensión sagrada de la naturaleza y de las dimensiones no racionales de la existencia, a través de la recuperación de un orden ancestral (Caicedo, 2009).

La orientación contemplativa se enmarca dentro de este contexto contemporáneo de, según Albert y Hernández (2014): "recuperación, revitalización y reconfiguración de las viejas formas de conocimiento trascendente vinculado a las antiguas filosofías y religiones, para ser transformadas en las nuevas creatividades culturales" (p. 275); y redención de las tradicionales técnicas de realización personal (Hotam y Wexler, 2014); en el cual se incorpora una reactualización de los ancestralmente probados saberes con la validación que la ciencia moderna ofrece. Este interés ha promovido la secularización de las prácticas de estas tradiciones, adquiriendo una forma amigable para el contexto de la cultura contemporánea occidental. Un ejemplo de ello es la técnica de meditación trascendental, la cual proveniente de la tradición hinduista, consta de varios volúmenes de información respecto de sus efectos en el ámbito clínico-hospitalario, educativo, organizacional y social-comunitario (Orme y Dillbeck, 2014); incluso instituciones de educación superior que incorporan sus prácticas en sus aulas comunes y ofrecen estudios de postgrado en la materia (Maharishi Foundation, 2015). También se puede contar con la técnica del Mindfulness-based stress reduction, la Mindfulness-based cognitive therapy y/o la Acceptance and commitment therapy, las cuales provenientes de la tradición budista, han mostrado ser eficaces en una serie de cuadros clínicos (Abbott et al., 2014; Piet, Wu y Zachariae, 2012). 


\section{Conclusiones: Una invitación a la autoexploración en el contexto educativo}

Se han expuesto algunos de los contextos que hacen posible la incorporación de la orientación contemplativa en la enseñanza y el aprendizaje en la práctica educativa, esperando generar interés en los profesionales de la educación por esta naciente orientación pedagógica y las prácticas que le acompañan. La educación contemplativa ofrece un amplio terreno para la integración de varios ámbitos esenciales para la ciencia pedagógica: investigación, integración e inclusividad, espiritualidad en el aula, moralidad, ética y cultivo de valores eudemónicos, transversalidad y competencias genéricas, incorporando así la dimensión del Ser en el aula. Además, esta orientación intenta alinearse con las tendencias en pedagogía contemporánea por un currículo basado en la evidencia (Detrich y Lewis, 2013), con el fin de garantizar el uso de estrategias pedagógicas validadas con un reconocible impacto en el estudiante y sus logros académicos. Por otra parte, se ha articulado también con los principios -ya citados- del procedimiento, los cuales sugieren un diseño curricular que concrete en la actividad teórico-práctica, el puente entre el currículo y las realidades concretas de acción, particularmente en el desarrollo de metodologías para el desarrollo socioafectivo. Finalmente, las prácticas que se originan desde esta orientación constituyen un aporte a una de las necesidades de mayor urgencia de los sistemas escolares por el buen trato y la convivencia escolar (Díaz y Sime, 2016); satisfaciendo varios de los lineamientos de las políticas públicas de los países de cambiar la mirada lógica instrumental, donde él y la estudiante son vistos como sujetos de problemas, a entender la convivencia escolar desde la formación continua del niño y niña para un sano y armonioso vivir en comunidad.

Recordando a Maslow (1969) y su concepto de desacralización, quien atisbó con exactitud hace varias décadas a las consecuencias que la expulsión de la subjetividad ha tenido en el sujeto contemporáneo, generándole un sentido de desconexión con la experiencia interna, con el cuerpo y emociones -y por extensión- con lo vivo y sus ecosistemas; la orientación contemplativa pretende constituirse en un aporte para volver a sacralizar la vida como respuesta a la obsolescencia en la que está envuelta la sociedad actual de consumo, ofreciendo una nueva lectura de las prácticas que validan a la subjetividad y formas de aproximación a la misma, como un aspecto esencial de la experiencia humana e imprescindible de incorporar en el proceso formativo de toda persona.

\section{Referencias}

Albert, R. y Hernández, M. (2014). Los movimientos psico-espirituales en la modernidad globalizada: Una mirada desde la ciudad de Valencia. Revista de Antropología Iberoamericana, 9(3), 273-296. https://doi.org/10.11156/aibr.090304

Álvarez, C. (2012). Los principios de procedimiento en el diseño curricular: Clave para la mejora de las relaciones teoría-práctica en educación. REICE. Revista Iberoamericana sobre Calidad, Eficacia y Cambio en Educación, 10(4), 21-36.

Abbott, R., Whear, R., Rodgers, L., Bethel, A., Thompson Coon, J., Kuyken, W., ... y Dickens, C. (2014). Effectiveness of mindfulness-based stress reduction and mindfulness based cognitive therapy in vascular disease: A systematic review and meta-analysis of 
randomised controlled trials. Journal of Psychosomatic Research, 76(5), 341-351. https://doi.org/10.1016/j.jpsychores.2014.02.012

Barbezat, D. y Bush, M. (2014). Contemplative practices in higher education. San Francisco, CA: Jossey Bass.

Bastián, M., Lerique, S., Adam, V., Franklin M., Schooler J. y Sackur, J. (2017). Language facilitates introspection: Verbal mind-wandering has privileged access to consciousness. Consciousness and Cognition, 49, 86-97. https://doi.org/10.1016/j.concog.2017.01.002

Berman, M. (1987). El reencantamiento del mundo. Santiago de Chile: Ed. Cuatro Vientos.

Bisquerra, R., y Pérez-Escoda, N. (2012). Educación emocional: Estrategias para su puesta en práctica. Avances en Supervisión Educativa, 16, 1-11.

Black, D., Milam, J. y Sussman, S. (2009). Sitting-meditation interventions among youth: A review of treatment efficacy. Pediatrics, 124(3), 532-541. https://doi.org/10.1542/peds.2008-3434

Brockman, R., Ciarrochi, J., Parker, P. y Kashdan, T. (2017). Emotion regulation strategies in daily life: Mindfulness, cognitive reappraisal and emotion suppression. Cognitive Behaviour Therapy, 46(2), 1-23. https://doi.org/10.1080/16506073.2016.1218926

Burack, C. (2014). Responding to the challenges of a contemplative curriculum. The Journal of Contemplative Inquiry, 1(1), 35-53.

Caicedo, A. (2009). Nuevos chamanismos nueva era. Universitas Humanística, 68, 15-32.

Cajigas, J. (2007). Estéticas de re(ex)sistencia. Por las sendas de la decolonización de la subjetividad. Nómadas, 26, 128-137.

Cardaciotto, L., Herbert, J., Forman, E., Moitra E. y Farrow, V. (2008). The assessment of present-moment awareness and acceptance: The Philadelphia mindfulness scale. Assessment, 15, 204-223. https://doi.org/10.1177/1073191107311467

Cárdenas, A., Soto, A., Dobbs, E. y Bobadilla, M. (2012). El saber pedagógico: Componentes para una reconceptualización. Educación y Educadores, 15(3), 479-496.

Casanova, M. (2012). El diseño curricular como factor de calidad educativa. REICE. Revista Iberoamericana sobre Calidad, Eficacia y Cambio en Educación, 10(4), 6-20.

Cejudo, J., López-Delgado, M. y Rubio, M. (2016). Inteligencia emocional y resiliencia: Su influencia en la satisfacción con la vida en estudiantes universitarios. Anuario de Psicología, 46, 51-57. https://doi.org/10.1016/j.anpsic.2016.07.001

Chano, J. (2012). An education in awareness: Recovering the heart of learning through contemplation. International Journal of Asian Social Science, 2(2), 106-110.

Chiesa, A., Calati, R. y Serretti, A. (2011). Does mindfulness training improve cognitive abilities? A systematic review of neuropsychological findings. Clinical Psychology Review, 31(3), 449464. https://doi.org/10.1016/j.cpr.2010.11.003

Damasio, A. (2000). Sentir lo que sucede. Cuerpo y emoción en la fábrica de la conciencia. Santiago de Chile: Ed. Andrés Bello.

Daugherty, A. (2014). From mindfulness to heartfulness: A journey of transformation through the science of embodiment. Bloomington, IN: Balboa Press.

Delors, J. (1996). La educación encierra un tesoro. Informe a la UNESCO de la Comisión internacional sobre la educación para el siglo XXI. Madrid: Santillana. 
Detrich, R. y Lewis, T. (2013). A decade of evidence-based education: Where are we and where do we need to go? Journal of Positive Behavior Interventions, 15(4), 14-220. https://doi.org/10.1177/1098300712460278

Dewey, J. (1910). How we think. Boston, MA: Heath \& CO.

Díaz, A., Carmona, A. y Nadia, C. (2010). La formación integral: Una mirada pedagógica desde los docentes. Colombian Applied Linguistics Journal, 12(1), 7-26.

Díaz, S. y Sime, L. (2016). Convivencia escolar: Una revisión de estudios de la educación básica en Latinoamérica. Revista Virtual Universidad Católica del Norte, 49, 125-145.

Duque, O. y Ramírez, F. (2012). El vacío existencial como desafío para los actuales educadores. Pre-Til, 10(27), 35-46.

Durlak, R., Weissberg, A., Dymnicki, R., Taylor, I. y Schellinger, K. (2011). The impact of enhancing students' social and emotional learning: A meta-analysis of school-based universal interventions. Child Development, 82, 405-432. https://doi.org/10.1111/j.14678624.2010.01564.x

Ergas, O. (2014). Mindfulness in education at the intersection of science, religion, and healing. Critical Studies in Education, 55(1), 58-72. https://doi.org/10.1080/17508487.2014.858643

Ergas, O. (2015a). The post-secular rhetoric of contemplative practice in the curriculum. En P. Wexler e Y. Hotam (Eds.), New social foundations for education: Education in post-secular society (pp. 107-130). Nueva York, NY: Peter Lang.

Ergas, O. (2015b). Educating the wandering mind: Pedagogical mechanisms of mindfulness for a curricular blind spot. Journal of Transformative Education, 14(2), 98-119. https://doi.org/10.1177/1541344615611258

Ferrer, J. y Sherman, J. (2009). The participatory turn: Spirituality, mysticism, religious studies. Albany, NY: State University of New York Press.

Flook, L., Goldberg, S., Pinger, L. y Davidson, R. (2015). Promoting prosocial behavior and selfregulatory skills in preschool children through a mindfulness-based kindness curriculum. Developmental Psychology, 51(1), 44-51. https://doi.org/10.1037/a0038256

Galante, J., Galante, I., Bekkers, M. y Gallacher, J. (2014). Effect of kindness-based meditation on health and well-being: A systematic review and meta-analysis. Journal of Consulting and Clinical Psychology, 82(6), 1101-1114. https://doi.org/10.1037/a0037249

García, B. y Klein, I. (2014). La construcción de ambientes educativos para la convivencia pacífica: el modelo pedagógico del programa SaludARTE. Sinéctica, 42, 1-13.

García, M. y Giménez, S. (2010). La inteligencia emocional y sus principales modelos: propuesta de un modelo integrador. Espiral. Cuadernos del Profesorado, 3(6), 43-52.

Gardner, H. (1987). La teoría de las inteligencias múltiples. Ciudad de México: Ed. Fondo de Cultura.

Garland, E. L., Hanley, A., Farb, y Froeliger, B. (2015). State mindfulness during meditation predicts enhanced cognitive reappraisal. Mindfulness, 6, 234-245. https://doi.org/10.1007/s12671-013-0250-6

Ghasemipour, Y., Robinson, J. y Ghorbani, N. (2013). Mindfulness and integrative selfknowledge: Relationships with health-related variables. International Journal of Psychology, 48(6), 1030-1037. http://doi.org/10.1080/00207594.2013.763948

Goleman, D. (1996). Inteligencia emocional. Barcelona: Kairós. 
González, M., Alegría, S., Barraza, D. y Villalobos, D. (2011). Construcción y validación preliminar de la escala de apertura al conocimiento y al cambio emocional en estudiantes universitarios. Terapia Psicológica, 29(2), 167-174. https://doi.org/10.4067/So71848082011000200003

Hart, T. (2004). Opening the contemplative mind in the classroom. Journal of Transformative Education, 2(1), 28-46. https://doi.org/10.1177/1541344603259311

Hotam, Y. y Wexler, P. (2014). Education in post-secular society. Critical Studies in Education, 55(1), 1-7. http://doi.org/10.1080/17508487.2014.857359

Immordino-Yang, M. y Faeth, M. (2010). Building smart students: A neuroscience perspective on the role of emotion and skilled intuition in learning. En D. A. Sousa (Ed.), Mind, brain, and education: Neuroscience implications for the classroom (pp. 66-81). Bloomington, IN: Solution Tree.

Jennings, P., Frank, J., Snowberg, K., Coccia, M. y Greenberg, M. (2013). Improving classroom learning environments by cultivating awareness and resilience in education (CARE): Results of a randomized controlled trial. School Psychology Quarterly, 28(4), 374-390. https://doi.org/10.1037/spq0000035

Kabat-Zinn, J. (2003). Mindfulness-based interventions in context: Past, present, and future. Clinical Psychology: Science $\mathcal{E}^{2} \quad$ Practice, $\quad 10(2), \quad 144-156$. https://doi.org/10.1093/clipsy.bpg016

Kirk, V., Fatola, C. y Romero, M. (2016). Systematic review of mindfulness induced neuroplasticity in adults: Potential areas of interest for the maturing adolescent brain. Journal of Childhood E Developmental Disorders, 2(1), 1-9. https://doi.org/ 10.4172/24721786.100016

Kok, B. y Singer, T. (2017). Effects of contemplative dyads on engagement and perceived social connectedness over 9 months of mental training: A randomized clinical trial. Psychiatry, 74(2), 126-134. https://doi.org/10.1001/jamapsychiatry.2016.3360

Langer, A., Ulloa, V., Cangas, A., Rojas, G. y Krause, M. (2015). Intervenciones basadas en mindfulness en educación secundaria: una revisión sistemática cualitativa. Estudios de Psicología, 36(3), 1-14. https://doi.org/10.1080/02 109395.2015.1078553

Langer, A., Schmidt, C., Aguilar-Parra, J., Cid, C. y Magni, A. (2017). Effects of a mindfulness intervention in Chilean high schoolers. Revista Médica de Chile, 145(4), 476-482. https://doi.org/10.4067/S0034-98872017000400008

Leria, F., Salgado, J., Almonte, I., Vega, M. y Véliz, M. (2016). Prácticas sociales en torno a la inclusión de la discapacidad sensorial en escuelas públicas de Copiapó de chile. Páginas de Educación, 9(1), 120-145. http://dx.doi.org/10.22235/pe.v9i1

Lynn, R. (2010) Mindfulness in social work education, social work education. The International Journal, 29(3), 289-304. https://doi.org/10.1080/02615470902930351

Maharishi Foundation. (2015). Transcendental meditation. Recuperado de http://www.tm.org/

Maslow, A. (1969). The psychology of Science: A reconnaissance. Nueva York, NY: Henry.

Mañas, I., Justo, C., Montoya, M. y Montoya, C. (2014). Educación consciente: Mindfulness (atención plena) en el ámbito educativo. Sevilla: Aconcagua Libros.

Martínez Ruíz, X. (2015). Atención consciente en la educación contemporánea: Una revolución silenciosa. Innovación Educativa, 15(67), 9-18. 
Matías, A. y Hernández, A. (2014). Positivismo, dialéctica materialista y fenomenología: Tres enfoques filosóficos del método científico y la investigación educativa. Actualidades Investigativas en Educación, 14(3), 1-20.

Mayer, J. y Salovey, P. (1997). What is emotional intelligence? En T. Salovey y M. Sluyter (Eds.), Emotional development and emotional intelligence: Implications for Educators (pp. 3-31). Nueva York, NY: Basic Books.

Mayer, J., Salovey, P. y Caruso, D. (2008). Emotional intelligence: New ability or eclectic traits? American Psychologist, 63(6), 503-517. https://doi.org/10.1037/0003-055x.63.6.503

Mazorco, G. (2010). Educación en crisis. Polis, 9(25), 269-288. https://dx.doi.org/10.4067/S07 18-65682010000100016

Meiklejohn, J., Phillips, C., Freedman, L., Griffin, M., Biegel, G., Roach, A., .., y Saltzman, A. (2012). Integrating mindfulness training into K-12 education: Fostering the resilience of teachers and students. Mindfulness, 3, 291-307. https://doi.org/10.1007/s12671-0120094-5

Murray, T. (2009). What is the integral in integral education? From progressive pedagogy to integral pedagogy. Integral Review, 5(1), 96-134.

Oña Cots, J. y García Gálvez, E. (2016). Proyecto escuela: Espacio de paz. Reflexiones sobre una experiencia en un centro educativo. REICE. Revista Iberoamericana sobre Calidad, Eficacia y Cambio en Educación, 14(2), 115-131. https://doi.org/10.15366/reice2016.14.2.007

Orme, D. y Dillbeck, M. (2014). Methodological concerns for meta-analyses of meditation. Psychological Bulletin, 140(2), 610-616. https://doi.org/10.1037/a0035896

Ortiz, T. (2009). Neurociencia y educación. Madrid: Alianza Editorial.

Palmer, P., Zajonc, A. y Scribner, M. (2010). The heart of higher education: A call to renewal, transforming the academy through collegial conversations ( $V$-XVII). San Francisco, CA: JosseyBass.

Páramo, P. y Hederich, C. (2014). Educación basada en la evidencia. Revista Colombiana de Educación, 66, 13-16.

Paymal, N. (2008). Pedagooogía 3000: Guía práctica para docentes, padres y uno mismo. Córdoba: Brujas.

Paymal, N. (2014). Pedagooogía 3000. Una pedagogía para el tercer milenio. La Paz: Editorial Ox La-Hun.

Peña, A., Mikolajczak, M. y Gross, J. (2015). Integrating emotion regulation and emotional intelligence traditions: A meta-analysis. Frontiers in Psychology, 6(160), 1-27. https://doi.org/10.3389/fpsyg.2015.00160

Piet, J., Wu, H. y Zachariae, R. (2012). The effect of mindfulness-based therapy on symptoms of anxiety and depression in adult cancer patients and survivors: A systematic review and meta-analysis. Journal of Consulting and Clinical Psychology, 80(6), 1007-1020. https://doi.org/10.1037/a0028329

Puebla, R. y Talma, M. (2011). Educación y neurociencias. La conexión que hace falta. Estudios Pedagógicos, 37(2), 379-388. https://doi.org/10.4067/S07 18-07052011000200023

Randal, C., Pratt, D. y Bucci, S. (2015). Mindfulness and self-esteem: A systematic review. Mindfulness, 6(6), 1-13. https://doi.org/10.1007/s12671-015-0407-6 
Remmers, C., Topolinski, S. y Koole, S. (2016). Why being mindful may have more benefits than you realize: Mindfulness improves both explicit and implicit mood regulation. Mindfulness, 7(4), 829-837. https://doi.org/10.1007/s12671-016-0520-1

Roeser, R. y Peck, S. (2009). An education in awareness: Self, motivation, and self-regulated learning in contemplative perspective. Educational Psychologist, 44(2), 119-136. https://doi.org/10.1080/00461520902832376

Schutte, S., Malouff, J. y Thorsteinsson, E. (2013). Increasing emotional intelligence through training: current status and future directions. The International Journal of Emotional Education, 5(1), 56-72.

Segovia, J. (2013). Un marco crítico de apoyo para ubicar y redireccionar experiencias innovadoras en educación: Comprensión y transformación. Tendencias Pedagógicas, 21, 928 .

Seli, P., Ralph, B., Risko, E., Schooler, J., Schacter, D. y Smilek, D. (2017). Intentionality and meta-awareness of mind wandering: Are they one and the same, or distinct dimensions? Psychonomic Bulletin and Review, 27, 9-11. https://doi.org/10.3758/s 13423-017-1249-0

Serrano, J. y Pons, R. (2011). El constructivismo hoy: Enfoques constructivistas en educación. Revista Electrónica de Investigación Educativa, 13(1), 1-23.

Shepherd, K., Coifman, K., Matt, L. y Fresco, D. (2016). Development of a self-distancing task and initial validation of responses. Psychological Assessment, 28(7), 841-855. https://doi.org/10.1037/pas0000297

Sternberg, R. J. (1985). Beyond IQ: A triarchic theory of human intelligence. Boston, MA: Cambridge University Press.

Taylor, C. (2007). A secular age. Boston, MA: The Belknap Press of Harvard University Press.

Teper, R., Segal, Z. y Inzlicht, M. (2013). Inside the mindful mind. How mindfulness enhances emotion regulation through improvements in executive control. Current Directions in Psychological Science, 22(6), 449-454. https://doi.org/10.1177/0963721413495869

Trousselard, M., Steiler, D., Claverie, D. y Canini, F. (2014). The history of mindfulness put to the test of current scientific data: Unresolved questions. Encephale, 40(6), 474-80. https://doi.org/10.1016/j.encep.2014.08.006

Thomas, G. y Atkinson, C. (2016). The effectiveness of a mindfulness-based intervention for children's attentional functioning. Educational \& Child Psychology, 33(1), 51-64.

Tomasino, B., Chiesa, A. y Fabbro, F. (2014). Disentangling the neural mechanisms involved in Hinduism and Buddhism-related meditations. Brain and Cognition, 90, 32-40. https://doi.org/10.1016/j.bandc.2014.03.013

UNESCO. (2008). La educación inclusiva: el camino hacia el futuro. Conclusiones y recomendaciones de la $48^{a}$ reunión de la Conferencia Internacional de Educación. Recuperado de http://www.ibe.unesco.org/

Vázquez, A. (2015). La cuestión del sujeto: Psicopatologías del yo y la transformación biopolítica de la subjetividad. Nómadas, 42, 53-75.

Waters, L., Barsky, A., Ridd, A. y Allen, K. (2014). Contemplative education: A systematic, evidence-based review of the effect of meditation interventions in schools. Educational Psychology Review, 27, 103-134. https://doi.org/10.1007/s10648-014-9258-2

Wang, Y. y Kong, F. (2014). The role of emotional intelligence in the impact of mindfulness on life satisfaction and mental distress. Social Indicators Research, 116, 843-852. https://doi.org/10.1007/s1 1205-013-0327-6 
Zajonc, A. (2013). Contemplative pedagogy: A quiet revolution in higher education. New Directions for Teaching and Learning, 134, 83-94. https://doi.org/10.1002/tl.20057

Zenner, C., Herrnleben, S. y Walach, H. (2014). Mindfulness-based interventions in schools. A systematic review and meta-analysis. Frontiers in Psychology, 5(603), 1-20. https://doi.org/10.3389/fpsyg.2014.00603

\section{Breve CV del autor}

\section{Francisco José Leria}

Psicólogo, Magister en Psicología clínica, Post-diplomado en Psicoterapia de Integración. Académico Universitario, profesor asistente (2014-2017); relator empresas (2011-2015); Psicólogo en hogar de menores (2009-2011); psicólogo centro social (20072009); psicoterapeuta práctica privada. Entre sus intereses académicos destacan: Educación Contemplativa, Educación emocional, Inclusividad e integración educativa. Otras áreas de interés son: estrés y factores protectores antes desastres socio-naturales, estrés laboral, motivación escolar. Actualmente está a cargo de un programa para el desarrollo de habilidades contemplativas para estudiantes de educación parvularia en práctica profesional. ORCID ID: 0000-0001-6326-2567. Email: francisco.leria@uda.cl 